Gut and Liver, Vol. 11, No. 1, January 2017, pp. 73-78

\title{
Underestimation of Smoking Rates in an East Asian Population with Crohn's Disease
}

Sung Wook Hwang ${ }^{1}$, Hyungil Seo ${ }^{1}$, Gwang-Un Kim ${ }^{1}$, Eun Mi Song ${ }^{1}$, Myeongsook Seo ${ }^{1}$, Sang Hyoung Park ${ }^{1}$, Eunja Kwon ${ }^{1}$, Ho-Su Lee ${ }^{2}$, Dong-Hoon Yang ${ }^{1}$, Kyung-Jo Kim ${ }^{1}$, Byong Duk Ye ${ }^{1}$, Jeong-Sik Byeon ${ }^{1}$, Seung-Jae Myung ${ }^{1}$, Jin-Ho Kim ${ }^{1}$, and Suk-Kyun Yang ${ }^{1}$

${ }^{1}$ Department of Gastroenterology and ${ }^{2}$ Health Screening and Promotion Center, Asan Medical Center, University of Ulsan College of Medicine, Seoul, Korea

\section{See editorial on page 7.}

Background/Aims: The reported rates of current smoking at the time of Crohn's disease (CD) diagnosis tend to be low in East Asian studies. However, we hypothesized that East Asian patients may be reluctant to disclose their smoking history, likely because of the influence of the Confucian culture. Methods: We prospectively re-evaluated the smoking status at diagnosis in 1,437 Korean CD patients whose smoking status had been reported in our previous study. Results: After re-evaluation, the current smokers at diagnosis increased from 388 patients (27.0\%) to 445 patients (31.0\%), indicating that $12.8 \%$ (57 of 445 patients) of the current smokers at diagnosis did not disclose their smoking status at their initial evaluation. The proportion of current smokers at diagnosis who had initially concealed their smoking status was significantly higher among the female patients $(29.7 \%, 11 / 37)$ compared with the male patients $(11.3 \%, 46 / 408)(p<0.005)$ and among the patients who were $\leq 18$ years old at diagnosis $(56.4 \%, 22 / 39)$ compared with the patients $>18$ years old at diagnosis $(8.6 \%, 35 / 406)(p<0.001)$. Conclusions: Subgroups of Korean CD patients, particularly young patients and female patients, are reluctant to disclose their smoking history. Therefore, the suggestion that smoking is not a risk factor for the development of $C D$ in East Asians should be made with caution. (Gut Liver 2017;11:73-78)

Key Words: Crohn disease; Smoking; Age at diagnosis; Sex

\section{INTRODUCTION}

Smoking is one of the most well-defined environmental risk factors for the development and progression of Crohn's disease (CD). ${ }^{1,2}$ However, most previous studies reported low rates (8.3\% to $16.6 \%$ ) of current smoking at CD diagnosis in East Asian patients, thereby suggesting a different role of smoking in the development of $\mathrm{CD}$ in this population. ${ }^{3-8}$ In contrast to these studies, two recent studies from China and Japan have reported relatively high rates (47\% and 38.5\%, respectively) of current smoking at CD diagnosis. ${ }^{9,10}$ The reason for this big difference in the rate of current smoking at CD diagnosis has yet to be adequately explained.

We have prospectively maintained the Asan inflammatory bowel disease (IBD) registry since 1997 and reported the longterm prognosis of $\mathrm{CD}$ in 2,043 Korean CD patients who are enrolled in the registry. ${ }^{11}$ In that study, the proportion of current smokers was 26.5\% at CD diagnosis. Although this figure was higher than reported in most East Asian studies, we felt that the smoking rate of our study was probably underestimated because Korean patients may be reluctant to disclose their smoking history due to the influence of Confucian culture. Therefore, we performed our present study to re-evaluate the smoking rate in Korean patients with CD. For this purpose, we reinterviewed the CD patients whose smoking status at diagnosis had been evaluated and reported in our previous study.

\section{MATERIALS AND METHODS}

\section{Study population}

We prospectively re-evaluated smoking status at $\mathrm{CD}$ diagnosis

\footnotetext{
Correspondence to: Suk-Kyun Yang

Department of Gastroenterology, Asan Medical Center, University of Ulsan College of Medicine, 88 Olympic-ro 43-gil, Songpa-gu, Seoul 05505, Korea

Tel: +82-2-3010-3901, Fax: +82-2-476-0824, E-mail: sky@amc.seoul.kr Received on April 13, 2016. Revised on May 15, 2016. Accepted on May 15, 2016. Published online October 13, 2016 pISSN 1976-2283 eISSN 2005-1212 https://doi.org/10.5009/gnl16194

@ This is an Open Access article distributed under the terms of the Creative Commons Attribution Non-Commercial License (http://creativecommons.org/licenses/by-nc/4.0) which permits unrestricted non-commercial use, distribution, and reproduction in any medium, provided the original work is properly cited.
} 
in Korean patients who had been seen between June 1989 and December 2012 at the Asan Medical Center, a tertiary university hospital in Seoul, South Korea, and whose smoking status had been evaluated in our previous study on the long-term prognosis of CD (Fig. 1). ${ }^{11}$ Re-evaluation was performed between January 1, 2015 and December 31, 2015. Among the 2,043 patients included in our previous study, 1,609 patients visited our outpatient clinic during the current study period. Of these 1,609 patients, smoking status at CD diagnosis was re-evaluated in 1,437 patients. The remaining 172 patients were excluded from this study because smoking history was not re-evaluated by attending physicians.

\section{Study design}

Baseline patient demographic and clinical information, including sex, date of birth, date of symptom onset, date of CD diagnosis, and smoking status at diagnosis, were retrieved from the Asan IBD registry. Smoking status at diagnosis was reevaluated at least once during the study period. After explaining to the patients about the importance of reporting accurate information to physicians, patients were asked again about their smoking status at diagnosis. Moreover, this process was performed in the absence of family members, such as parents and spouses, because patient responses might be influenced by their presence. The definition of smoking status at diagnosis in our current study did not differ from our previous study. ${ }^{11,12}$ Never smokers were defined as those who had a history of smoking fewer than 100 cigarettes in their entire life. Current smokers were defined as those who had a history of smoking at least 100 cigarettes in their entire life and had continued smoking within 6 months prior to CD diagnosis. Ex-smokers were defined as those who had a history of smoking at least 100 cigarettes in their entire life and had completely stopped smoking for at least 6 months prior to CD diagnosis. The factors associated with changes in smoking status at diagnosis as a result of re-evaluation were analyzed.

The Ethics Committee of Asan Medical Center approved the study protocol, and written informed consent was obtained from all patients.

\section{Statistical analysis}

Continuous variables were expressed as the median and interquartile range (IQR), and categorical variables were expressed as a number and percentage (\%). The Mann-Whitney U test was used to compare continuous variables between groups, and the chi-square or Fisher exact test was used to compare categorical

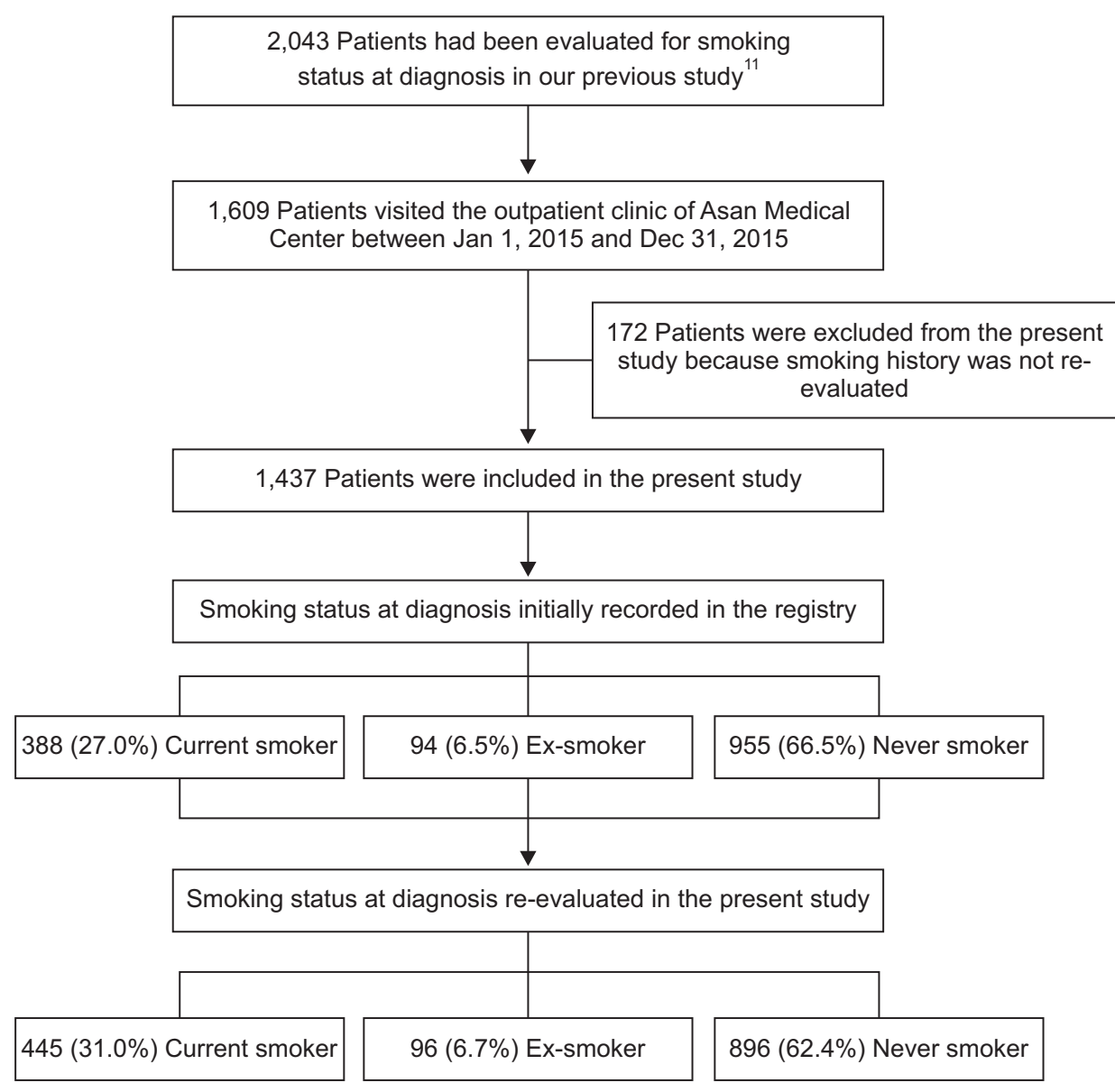

Fig. 1. Flow chart of the recruitment of patients with Crohn's disease and the re-evaluation of smoking status at diagnosis. 
variables. These analyses were performed using the IBM SPSS version 21.0 for Windows (IBM Corp., Armonk, NY, USA). A $\mathrm{p}<0.05$ was considered as the level of significance.

\section{RESULTS}

Of the 1,437 patients included in our study, 1,021 patients (71.1\%) were male and 416 patients (28.9\%) were female, yielding a male-to-female ratio of 2.45:1. The median age at CD diagnosis was 23 years (range, 9 to 75 years). Smoking status at diagnosis initially recorded in the registry for the 1,437 study patients was as follows: current smoker in 388 patients (27.0\%), ex-smoker in 94 patients (6.5\%), and never smoker in 955 patients (66.5\%) (Fig. 1). These rates were not different from those among all 2,043 patients reported in our previous study. ${ }^{11}$

Smoking status at diagnosis was re-evaluated after at least 2 years had passed from the initial evaluation, and the median interval between evaluations was 6.0 years (IQR, 3.0 to 10.0 years). After re-evaluation, smoking status at diagnosis changed from never smoker to current smoker in 41 patients and to exsmoker in 18 patients. Also, smoking status changed from exsmoker to current smoker in 16 patients. Consequently, the number of current smokers at diagnosis increased to 445 patients (31.0\%), and the number of ex-smokers increased to 96 patients (6.7\%), whereas the number of never smokers decreased to 896 patients (62.4\%) (Fig. 1). This indicates that 12.8\% (57 of 445 patients) of current smokers at diagnosis did not disclose their smoking status at their initial evaluation.

When analyzed by sex, the proportion of current smokers who had initially concealed their smoking status was higher in female patients $(29.7 \%, 11$ of 37 patients) than male patients (11.3\%, 46 of 408 patients) ( $\mathrm{p}<0.005)$. When analyzed by age, the proportion of current smokers who had initially concealed their smoking status was higher in patients 18 years or younger at diagnosis (56.4\%, 22 of 39 patients) than in patients older than 18 years at diagnosis $(8.6 \%, 35$ of 406 patients) $(\mathrm{p}<0.001)$ (Table 1). Among patients older than 18 years at diagnosis, the proportion of current smokers who had initially hidden their smoking status was $11.1 \%$ (30 of 271 patients) in patients 30 years or younger at diagnosis in comparison to $3.7 \%$ (five of 135 patients) of patients older than 30 years at diagnosis $(\mathrm{p}<0.05)$.

\section{DISCUSSION}

To the best of our knowledge, our present study is the first to re-evaluate smoking status at CD diagnosis in the same group of patients. In Korea, due to the influence of Confucius on cultural practices, it is considered taboo for young people to smoke in front of their elders and for women to smoke in public. In addition, considering the fact that obeying parents and elders is one of the important values of the Confucian culture, it is highly possible that the patients do not disclose their smoking status in East Asian countries. The smoking rate of women is much lower than that of men in Korea (41.4\% in adult men vs $5.7 \%$ in adult women), ${ }^{13}$ and the smoking rate of adolescence in Korea is 9.7\% according to the Korean Centers for Disease Control (CDC) database. ${ }^{14}$ Therefore, we hypothesized that some Korean patients, especially young patients and female patients, would be reluctant to honestly reveal their smoking history to their doctors. We also hypothesized that patients would disclose the correct information regarding their smoking status at diagnosis if (1) they were older and more familiar with their attending physicians, (2) they were asked about their smoking history in the absence of family members, and (3) they realized the importance of this information to their medical treatment. Our present study demonstrated that the rate of current smoking at diagnosis was underestimated in our previous cohort of Korean CD patients, especially in young patients and female patients, thereby confirming our hypothesis.

Even after re-evaluation, however, the proportion of current smokers at diagnosis increased by only $4.0 \%$ and is apparently still low in comparison with Western CD patients. ${ }^{15-19}$ Western population-based studies reported the smoking status at CD diagnosis as follows: $30 \%$ to $40 \%$ current smokers, $7 \%$ to $24 \%$ ex-smokers, and 38\% to 52\% never smokers. The apparently lower rate of smoking in our Korean CD patients has several possible explanations. First, the median age at diagnosis in our study (23 years) was lower than that in Western studies (30 to 34 years). ${ }^{15-20}$ More than $25 \%$ of our patients were diagnosed at

Table 1. Changes in Smoking Status at the Time of Diagnosis of Crohn's Disease as a Result of the Re-Evaluation of Smoking Status according to Sex and Age at Diagnosis

\begin{tabular}{|c|c|c|c|c|c|}
\hline \multirow[b]{2}{*}{ Sex } & \multirow[b]{2}{*}{ Age at diagnosis, yr } & \multirow[b]{2}{*}{ No. of patients } & \multicolumn{2}{|c|}{ Current smoker at diagnosis } & \multirow{2}{*}{$\begin{array}{l}\text { Proportion of initial misclassifications in } \\
\text { current smokers at diagnosis, } \%(\mathrm{n} / \mathrm{N})\end{array}$} \\
\hline & & & $\begin{array}{l}\text { Before re-evaluation, } \\
\mathrm{n}(\%)\end{array}$ & $\begin{array}{l}\text { After re-evaluation, } \\
\text { n (\%) }\end{array}$ & \\
\hline \multirow[t]{2}{*}{ Female } & $\leq 18$ & 109 & $1(0.9)$ & $3(2.8)$ & $66.7(2 / 3)$ \\
\hline & $>18$ & 307 & $25(8.1)$ & $34(11.1)$ & $26.5(9 / 34)$ \\
\hline \multirow[t]{2}{*}{ Male } & $\leq 18$ & 262 & $16(6.1)$ & $36(13.7)$ & $55.6(20 / 36)$ \\
\hline & $>18$ & 759 & $346(45.6)$ & $372(49.0)$ & $7.0(26 / 372)$ \\
\hline
\end{tabular}


the age of 18 years or younger, thus lowering the smoking rate in our series. Second, as mentioned above, the smoking rate of women is much lower than that of men in Korea, which lowers the overall smoking rate. ${ }^{13}$ In fact, the rate of current smoking in Korean male CD patients older than 18 years at diagnosis was 49.0\% (372 of 759 patients), which was higher than the current smoking rate of $41.4 \%$ in the Korean adult male population. Similarly, the rate of current smoking in Korean female CD patients older than 18 years of age at diagnosis is 11.1\% (34 of 307 patients), which is higher than the current smoking rate of 5.7\% in the Korean adult female population. Although this comparison is crude, it suggests that smoking may also play a role in the development of $\mathrm{CD}$ in Koreans. In contrast, the rate of current smoking at diagnosis in our male and female patients 18 years or younger at diagnosis (13.7\% and 2.8\%, respectively) in the present study was not higher than the current smoking rate in the Korean males and females 18 years or younger (14.4\% and $4.6 \%$ according to the Korean CDC, respectively). ${ }^{14}$ This raises the possibility that some young patients may still have been withholding their true smoking status at CD diagnosis despite our re-evaluation.

Previous East Asian studies have suggested that the role of smoking in the development of CD may differ between different ethnic groups due to genetic heterogeneities and that, given the low rate of current smoking at diagnosis in East Asians, smoking is not a significant risk factor for CD in this population. ${ }^{3,8,21,22}$ According to the findings of previous Israeli studies, there is no association between smoking and CD in the Jewish population. ${ }^{23-26}$ This unusual lack of association is likely caused by the masking of smoking effects by the strong genetic predisposition for $\mathrm{CD}$ in this ethnic group. However, this is not the case in East Asians. Moreover, previous studies have demonstrated an association between smoking and intestinal resection in East Asian patients with $\mathrm{CD} .{ }^{4,5,10}$ Considering that two recent studies from China and Japan reported relatively high rates (47\% and 38.5\%, respectively) of current smoking at CD diagnosis, ${ }^{9,10}$ and that our study demonstrated a high rate of current smoking at diagnosis in Korean adult male patients, the smoking status at diagnosis should be cautiously evaluated and interpreted in East Asians patients.

Besides deliberate false replies from patients, various factors may cause the underestimation of smoking status at diagnosis. First, some patients who quit smoking after symptom onset but before $\mathrm{CD}$ diagnosis may just reply that they do not smoke if the question at the time of CD diagnosis is "Do you smoke?" instead of "Have you ever smoked?" These patients may be falsely classified as never smokers at CD diagnosis, but they should be classified as ex-smokers or current smokers at $\mathrm{CD}$ diagnosis depending on the interval between the cessation of smoking and $\mathrm{CD}$ diagnosis. In our current study, 80 patients quit smoking after the onset of symptoms and before the diagnosis of $\mathrm{CD}$. Of these 80 cases, 50 patients ceased smoking within 6 months before $\mathrm{CD}$ diagnosis (data not shown). If these 50 patients had been falsely classified as never smokers due to inadequate history taking, the proportion of current smokers at diagnosis would have falsely appeared as $27.5 \%$ rather than $31.0 \%$. Second, many patients quit smoking during the disease course, especially just after $\mathrm{CD}$ diagnosis. These patients may be falsely classified as never smokers or ex-smokers at CD diagnosis if smoking history is inappropriately taken after the cessation of smoking. In our study, 68 current smokers at diagnosis had already quit smoking when they were referred to our hospital (data not shown). If these patients had been falsely classified as never smokers or ex-smokers at diagnosis due to inadequate historytaking at their first visit, the proportion of current smokers at diagnosis would have falsely appeared as $26.2 \%$ rather than $31.0 \%$. Third, information on smoking status may be unavailable for some patients, even in prospective studies. ${ }^{3,27}$ If these patients are mistakenly classified as never smokers, the smoking rate may inaccurately decrease. Taken together, smoking status at diagnosis should be cautiously evaluated before concluding that smoking is not a risk factor for the development of CD in East Asians.

Our study had some limitations. First, although we tried to be as accurate as possible when re-evaluating the smoking status at diagnosis, there was still a possibility that the result was underestimated because our assessment only relied on patient responses. Nicotine testing at the time of $\mathrm{CD}$ diagnosis may be necessary for the accurate evaluation of smoking status at diagnosis. ${ }^{28}$ Second, the patient's memory of smoking status at diagnosis may be unclear in some cases because of the time interval between CD diagnosis and re-evaluation of smoking status. However, we believe this was unlikely because most patients were educated at the time, or in the early days following $\mathrm{CD}$ diagnosis, about the deleterious effects of smoking on this disease. Third, although our results suggest that smoking may play a role in the development of CD in Koreans, like in Westerners, it is still premature to conclude from our current findings that smoking is a definite risk factor for the development of $\mathrm{CD}$ in East Asians.

In conclusion, some Korean CD patients, especially young patients and female patients, are reluctant to disclose their smoking history. Therefore, we should be careful to obtain correct information about smoking history, and the suggestion that smoking is not a risk factor for the development of CD in East Asians who have a similar cultural background as Koreans should be made with caution.

\section{CONFLICTS OF INTEREST}

Suk-Kyun Yang received a research grant from Janssen Korea Ltd., but this grant was not related to the topic of the current study. The remaining authors have no competing interests to declare. 


\section{ACKNOWLEDGEMENTS}

This study was supported by a grant from the Korean Health Technology R\&D Project, Ministry of Health \& Welfare, Republic of Korea (A120176).

\section{REFERENCES}

1. Nunes T, Etchevers MJ, Domènech E, et al. Smoking does influence disease behaviour and impacts the need for therapy in Crohn's disease in the biologic era. Aliment Pharmacol Ther 2013;38:752-760.

2. Lakatos PL, Vegh Z, Lovasz BD, et al. Is current smoking still an important environmental factor in inflammatory bowel diseases? Results from a population-based incident cohort. Inflamm Bowel Dis 2013;19:1010-1017.

3. Prideaux L, Kamm MA, De Cruz P, et al. Comparison of clinical characteristics and management of inflammatory bowel disease in Hong Kong versus Melbourne. J Gastroenterol Hepatol 2012;27:919-927.

4. Moon CM, Park DI, Kim ER, et al. Clinical features and predictors of clinical outcomes in Korean patients with Crohn's disease: a Korean association for the study of intestinal diseases multicenter study. J Gastroenterol Hepatol 2014;29:74-82.

5. Song XM, Gao X, Li MZ, et al. Clinical features and risk factors for primary surgery in 205 patients with Crohn's disease: analysis of a South China cohort. Dis Colon Rectum 2011;54:1147-1154.

6. Li Y, Ren J, Wang G, et al. Diagnostic delay in Crohn's disease is associated with increased rate of abdominal surgery: a retrospective study in Chinese patients. Dig Liver Dis 2015;47:544-548.

7. Pandey A, Salazar E, Kong CS, et al. Risk of Major Abdominal Surgery in an Asian Population-based Crohn's Disease Cohort. Inflamm Bowel Dis 2015;21:2625-2633.

8. Ng SC, Tang W, Leong RW, et al. Environmental risk factors in inflammatory bowel disease: a population-based case-control study in Asia-Pacific. Gut 2015;64:1063-1071.

9. Zhao J, Ng SC, Lei Y, et al. First prospective, population-based inflammatory bowel disease incidence study in mainland of China: the emergence of "Western" disease. Inflamm Bowel Dis 2013;19: 1839-1845.

10. Sato Y, Matsui T, Yano Y, et al. Long-term course of Crohn's disease in Japan: incidence of complications, cumulative rate of initial surgery, and risk factors at diagnosis for initial surgery. $\mathrm{J}$ Gastroenterol Hepatol 2015;30:1713-1719.

11. Park SH, Yang SK, Park SK, et al. Long-term prognosis of Crohn's disease and its temporal change between 1981 and 2012: a hospital-based cohort study from Korea. Inflamm Bowel Dis 2014;20: 488-494.

12. Leung Y, Kaplan GG, Rioux KP, et al. Assessment of variables associated with smoking cessation in Crohn's disease. Dig Dis Sci 2012;57:1026-1032.

13. Health behaviors and chronic disease statistics in 2015 [Internet].
Cheongju: Centers for Disease Control \& Prevention; c2015 [cited 2016 Jun 21]. Available from: http://www.cdc.go.kr/CDC/notice/ CdcKrIntro0504.jsp?menuIds=HOME001-MNU1154-MNU0004MNU0110\&cid $=65024$.

14. Reports on the Korea youth risk behavior web-based survey [Internet]. Cheongju: Centers for Disease Control \& Prevention; c2015 [cited 2016 Jun 21]. Available from: http://www.cdc.go.kr/ CDC/intro/CdcKrIntro0201.jsp?menuIds=HOME001-MNU1154MNU0005-MNU0011\&cid=66275.

15. Vester-Andersen MK, Prosberg MV, Jess T, et al. Disease course and surgery rates in inflammatory bowel disease: a populationbased, 7-year follow-up study in the era of immunomodulating therapy. Am J Gastroenterol 2014;109:705-714.

16. Nuij VJ, Zelinkova Z, Rijk MC, et al. Phenotype of inflammatory bowel disease at diagnosis in the Netherlands: a population-based inception cohort study (the Delta Cohort). Inflamm Bowel Dis 2013;19:2215-2222.

17. Chatu S, Saxena S, Subramanian V, et al. The impact of timing and duration of thiopurine treatment on first intestinal resection in Crohn's disease: national UK population-based study 1989-2010. Am J Gastroenterol 2014;109:409-416.

18. Thia KT, Sandborn WJ, Harmsen WS, Zinsmeister AR, Loftus EV Jr. Risk factors associated with progression to intestinal complications of Crohn's disease in a population-based cohort. Gastroenterology 2010;139:1147-1155.

19. Burisch J, Pedersen N, Čuković-Čavka S, et al. East-West gradient in the incidence of inflammatory bowel disease in Europe: the ECCO-EpiCom inception cohort. Gut 2014;63:588-597.

20. Vester-Andersen MK, Vind I, Prosberg MV, et al. Hospitalisation, surgical and medical recurrence rates in inflammatory bowel disease 2003-2011: a Danish population-based cohort study. J Crohns Colitis 2014;8:1675-1683.

21. Ng SC. Emerging leadership lecture: inflammatory bowel disease in Asia: emergence of a "Western" disease. J Gastroenterol Hepatol 2015;30:440-445.

22. Prideaux L, Kamm MA, De Cruz PP, Chan FK, Ng SC. Inflammatory bowel disease in Asia: a systematic review. J Gastroenterol Hepatol 2012;27:1266-1280.

23. Fidder HH, Avidan B, Lahav M, Bar-Meir S, Chowers Y. Clinical and demographic characterization of Jewish Crohn's disease patients in Israel. J Clin Gastroenterol 2003;36:8-12.

24. Reif S, Lavy A, Keter D, et al. Lack of association between smoking and Crohn's disease but the usual association with ulcerative colitis in Jewish patients in Israel: a multicenter study. Am J Gastroenterol 2000;95:474-478.

25. Fich A, Eliakim R, Sperber AD, Carel RS, Rachmilewitz D. The association between smoking and inflammatory bowel disease among israeli jewish patients. Inflamm Bowel Dis 1997;3:6-9.

26. Reif S, Klein I, Arber N, Gilat T. Lack of association between smoking and inflammatory bowel disease in Jewish patients in Israel. Gastroenterology 1995;108:1683-1687.

27. Ng SC, Tang W, Ching JY, et al. Incidence and phenotype of 
78 Gut and Liver, Vol. 11, No. 1, January 2017

inflammatory bowel disease based on results from the AsiaPacific Crohn's and colitis epidemiology study. Gastroenterology 2013;145:158-165.e2.
28. Nunes T, Etchevers MJ, Merino 0, et al. High smoking cessation rate in Crohn's disease patients after physician advice: the TABACROHN Study. J Crohns Colitis 2013;7:202-207. 